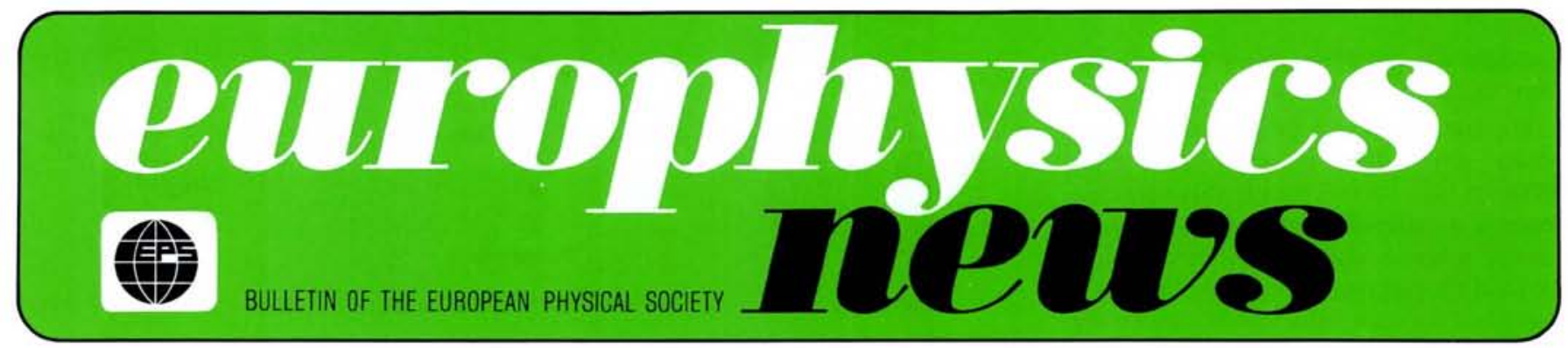

J.A.

Volume 12

Number 2

February 1981

The 1980 Nobel Prize in physics was awarded to Professor James W. Cronin, Enrico Fermi Institute, and Professor Val L. Fitch, Princeton University, for "the discovery of violations of fundamental symmetry principles in the decay of neutral K-mesons".

Symmetries are often associated with regularity, beauty and perfection and as such they have played and play today an important role in art, music, human way of thinking and culture. Symmetries have aroused admiration, respect and fear. In some societies the symmetric patterns were deliberately "broken" slightly so as not to annoy the envious gods whose reactions could lead to disaster. Greek mythology tells of the fate of the young girl Arakne who excelled the gods in weaving the most perfect tapestries. The goddess of art and wisdom, Athene, punished Arakhne by transforming her into a spider.

In physics, symmetries and invariance principles have played a most important role, especially during our century. Groping in the dark, to decipher the structure of the laws of Nature, physicists have invented symmetries so that they may serve as a starting point and as guiding principles. Their intuition - or prejudice - leads to the belief that the laws of Nature are

\section{Contents}

Broken Symmetries

HEPP: Where Do We Stand?

Quantum Electronics:

Changes in Emphasis

Interaction Between Physics and Society

Physics Teaching at Secondary School Level in Europe

Physics in One Dimension

New Individual Ordinary

Members

Society News

\title{
Broken Symmetries
}

\section{Jarlskog, Bergen}

necessarily simple and beautiful and exhibit a great deal of regularity. Often, however, the intuition goes wrong and a symmetry which was thought to be indispensable turns out to be "broken". This makes the symmetry even more interesting and useful as it provides the crack which can lead to a deeper penetration into how's and why's. Nobel Prizes in physics bear ample witness to this as can be seen from a brief look at recent history.

Taking the past 25 years, only: in 1957, T.D. Lee and C.N. Yang were honoured for their work on the violation of the reflection symmetry, in 1963 E.P. Wigner for "... the discovery and applications of fundamental symmetry principles". SU(3)-symmetry was at the centre of the 1969 award to M. Gell-Mann, and ten years later S. Glashow, A. Salam and S. Weinberg received the Prize for their unified theory of weak and electromagnetic interactions based on the concept of spontaneously broken local symmetries.

\section{Right Hand Left Hand}

Symmetries relevant to the 1980 Nobel Prize are space reflection (or parity P), charge conjugation $\mathrm{C}$, and time reversal. Until 25 years ago, a physicist would have been considered stupid if she or he had claimed that Nature was not left-right symmetric. It was taken for granted that any physical process and its mirror image were equivalent. The laws of classical physics are invariant under parity. Atomic physics, by respecting the left-right symmetry (Laporte's selection rules), fertilized this useful concept, so why should interactions of elementary particles also not be left-right symmetric? It was the observation of the decay modes of charged $\mathrm{K}$-mesons,

$$
\mathrm{K} \rightarrow \pi+\pi, \mathrm{K}-\pi+\pi+\pi,
$$

which led Lee and Yang to examine the evidence for left-right symmetry in particle physics. They found that there was no evidence for parity conservation in weak interactions and suggested experiments which could unambiguously settle the issue. The

Professors James W. Cronin (left) and Val L. Fitch in Stockholm in December of last year (Courtesy of Prof. Bengt Nagel of the Swedish Royal Academy of Sciences.)

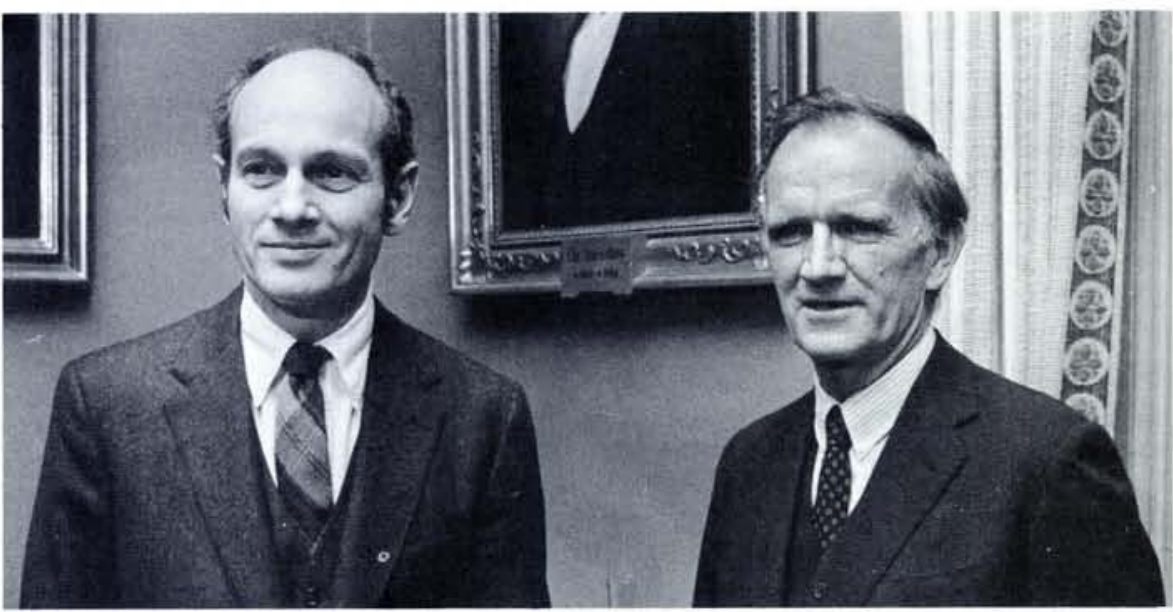


outcome was dazzling: weak interactions were found to violate the holy principle.

The concept of charge conjugation originated from the Dirac equation (Nobel Prize 1933). It was known that in this equation there is a symmetry between the positive energy solution which describes the particle, and the negative energy solution which describes the hole, later identified with the antiparticle. This particle-antiparticle symmetry was formalized by Pauli in 1936, through the operation of charge conjugation. The hypothesis of C-symmetry implies that any physical process and its $\mathrm{C}$-image, where each particle is replaced by its "antiparticle", are equivalent. The C-symmetry seemed a very reasonable hypothesis. Why should nature treat particles and antiparticles differently? However with the fall of parity, C-symmetry was also invalidated.

A simple example of the mirror symmetry and $\mathrm{C}$-symmetry is provided by the pion decay $\pi^{+} \rightarrow \mu^{+}+v$, shown in Fig. 1 . The pion is considered to decay at rest, resulting in the muon and the neutrino flying off in opposite directions. The mirror image, denoted by $\mathrm{P}$-image, of this reaction is obtained by reversing the directions of motion, leaving the spins unchanged. Thus the helicity or handedness of any particle is reversed in the mirror. So if parity were good, the reaction and its mirror image must happen with equal probabilities. They do not. In the laboratory, the neutrino is found to be left-handed. Its mirror image, the right-handed neutrino, has not been seen so far, either because it is much suppressed or because it does not exist at all.

Physicists could not believe that in weak interactions Nature distinguishes left from right and does not treat particles and antiparticles equivalently. For example, Pauli exclaimed that he did not believe that "God is a weak left-hander". However, he also realized that "between believing and knowing, there is a difference, and in the last, such questions must be decided experimentally". When Pauli was informed that parity violation in weak interactions was empirically established, he found consolation by noting that "God has in his left hand $\left(e^{-}, v\right)$, in his right hand $\left(e^{-}, \bar{v}\right)^{\prime \prime}$. In other words, the particle is in the left hand and its $\mathrm{C}$-image is in the right hand. This amounts to an assumption of CP-invariance as was proposed by Landau, Lee and Yang, and Salam in order to restore a kind of ambidexterity to the laws of Nature.

A CP-mirror changes a particle into an "antiparticle" and reverses the direction of motion. The CP-image of $\pi^{+} \rightarrow \mu^{+}+v$ with a left-handed neutrino is $\pi^{-} \rightarrow \mu^{-}+\bar{v}$ with a right-handed antineutrino. The process and its mirror image (Fig. 2) indeed occur with the same probability, which gave comforting support to another prejudice, namely that time reversal invariance should hold in Nature.

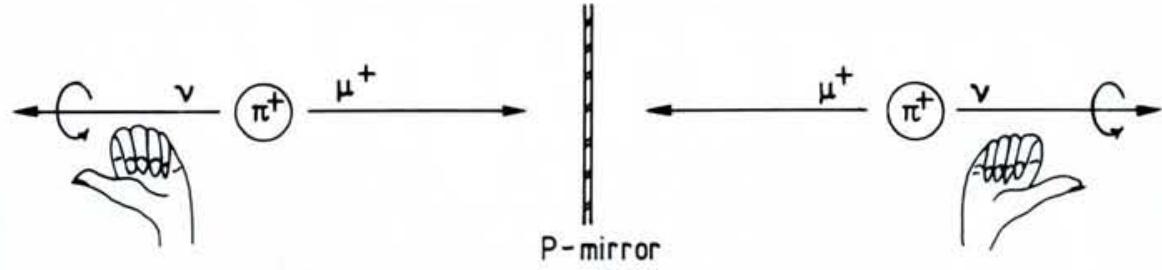

Fig. 1 - The decay of a positive pion with a left-handed neutrino and its mirror image. The mirror process does not occur in Nature.

\section{Time Reversal Invariance}

The laws of classical physics are formally invariant when the arrow of time is reversed. Time reversal is a good symmetry and a useful concept in atomic physics (see, e.g. Kramers' degeneracy). In particle physics, a more appropriate interpretation of this symmetry would have been Lüders' "Umkehr der Bewegungsrichtung" or (direction of) motion reversal symmetry. The T-image of an event is one in which the initial and final states are interchanged and, in addition, all momenta and spins are reversed. There is a fundamental theorem in physics called the CPT theorem which states that symmetry CPT. The theorem rests on a few reasonable and seemingly fundamental assumptions.

Perhaps some day the theorem will turn out to be contradicted by experiment. Some will weep in sorrow over the lost beauty; some will celebrate a Nobel Prize. At our present stage of understanding, the CPT symmetry is very fundamental. It tells us that each particle has an antiparticle, the antiparticle being the CPT image of the particle. The particle and its antiparticle have the same masses and lifetimes, by CPT symmetry. From CPT symmetry follows also that the T symmetry and CP symmetry are either both good or both violated. and CP-symmetry appeared to be respected. In that year the 1980 Nobel Laureates J.W. Cronin and V.L. Fitch at Princeton University, together with two collaborators J.H. Christenson and R. Turlay announced the astounding discovery of $\mathrm{CP}$-violation in the two pion decay mode of the long-lived neutral $\mathrm{K}$-meson, $\mathrm{K}_{\mathrm{L}} \rightarrow \pi^{+}+\pi^{-}$. There are two neutral kaons, the short-lived $\mathrm{K}_{\mathrm{S}}$ the laws of Nature respect the product

Up to 1964 the time reversal symmetry T and the long-lived $K_{L}$. These particles were thought to be eigenstates of $\mathrm{CP}$, i.e. one of them was its own CP-image, and the wave function of the other, reflected in the CP. mirror, merely changed in sign. It was then concluded that only one of the neutral kaons could decay into two pions, and as $\mathrm{K}_{\mathrm{S}} \rightarrow \pi+\pi$ occurred, the decay $\mathrm{K}_{\mathrm{L}} \rightarrow \pi^{+}+\pi^{-}$ was forbidden.

\section{The Crucial Experiment}

The experiment which led to the 1980 haven National Laboratory where a beam of $K_{L}$, produced in collisions of $30 \mathrm{GeV}$ protons with an internal Be target, was allowed to decay inside a helium bag (Fig. 3).

Two detectors, placed symmetrical with respect to the beam, could register decay products with positive and negative charges. A vital issue in looking for the forbidden $\mathrm{K}_{L} \rightarrow \pi^{+}+\pi^{-}$was how to isolate the effect, if any, from the huge background: previous experiments had established an upper limit of $1 / 400$ for the branching ratio of that particular decay mode. The Princeton group employed an ingenious device. Pieces of tungsten were introduced successively along the path of the $\mathrm{K}_{\mathrm{L}}$-beam in each of which beams of $\mathrm{K}_{\mathrm{S}}$ were generated with the same momentum as $K_{L}$ (coherent regeneration).

A large fraction of $\mathrm{K}_{\mathrm{S}}$ particles decay rapidly into two charged pions and these were detected. In this way the unknown decay mode $K_{L} \rightarrow \pi^{+} \pi^{-}$was related to the known $\mathrm{K}_{\mathrm{S}} \rightarrow \pi^{+} \pi^{-}$. The Princeton group found a clear signal for the forbidden decay, with a probability against $K_{L} \rightarrow$ all charged modes, of $(2.0 \pm 0.4) 10^{-3}$.

This experiment (and the numerous ones which followed) established unequivocally that $\mathrm{CP}$-invariance is violated: the $\mathrm{CP}$ Nobel Prize was performed at the Brook-

Fig. 2 - The CP-image of a left-handed neutrino is a right-handed antineutrino. Here, the event and its mirror image occur with equal probabilities.
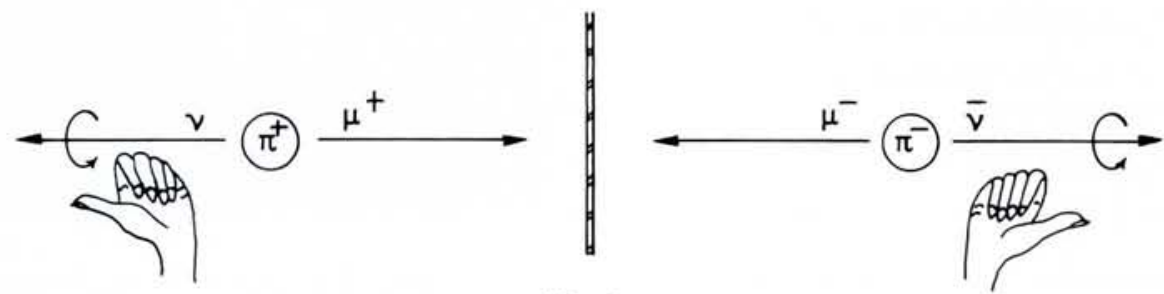

CP-mirror 
image of $K_{L}$ is not a "pure" particle with well-defined mass and lifetime. The $\mathrm{CP}$ impurity, $\varepsilon$, found by the Princeton group, in the wave function of $K_{L}$ was assessed as $|\varepsilon|=2.3 \times 10^{-3}$ which is in impressive agreement with the present world average of $\varepsilon=(2.28 \pm 0.03) 10^{-3}$.

To date CP-violation has been seen only in the decay modes of the $\mathrm{K}_{\mathrm{L}}, \mathrm{K}_{\mathrm{L}} \rightarrow \pi \pi$, $\mathrm{K}_{\mathrm{L}} \rightarrow \pi \mathrm{ev}, \mathrm{K}_{\mathrm{L}} \rightarrow \pi \mu v$, all of which are compatible with the assumption that the wave function of $\mathrm{K}_{\mathrm{L}}$ has a tiny $\mathrm{CP}$-impure component, governed uniquely by the parameter $\varepsilon$.

With CP-violation, the "ambidexterity" of Nature is more subtle than had been supposed. Had Pauli been alive he would have had to say that God has a particle in his left hand and its CT-image in his right hand.

\section{Consequences of CP Violation}

Why is CP violated? Why is the violation small? Why does it show up exclusively in $\mathrm{K}$ decay? Why do only weak interactions violate $\mathrm{CP}$ ? These are questions which, since, 1964, have been puzzling particle physicists and exerting a major influence on further developments in the field.

The first question is perhaps wrongly posed: we should not ask why a symmetry is violated, but why it is respected. In 1973, Kobayashi and Maskawa studied CP-violation in the context of unified theories. They found that in the presently standard theory of weak and electromagnetic interactions (Nobel Prize in 1979), CP is conserved if there are only four quarks, which was the situation at that time, whereas at least two more quarks are needed to account for the observed CP-violation. The fifth quark was revealed in 1977; the sixth has yet to be be found. In the standard model with six quarks, the phenomenon of CP-violation takes place in weak interactions of all quarks. However, the effects are small and experimentally hard to detect. There is nothing special about neutral kaons except that they are the lightest mesons which decay by weak interactions, and consequently live a relatively long time and are amenable to experimentation.

Several other mechanisms for CP-violation have also been proposed: Wolfenstein's superweak theory, spontaneous $\mathrm{CP}$ violation à la T.D. Lee and S. Weinberg, etc. With the dazzling successes of the standard model (see page 4) the alternative explanations are less fashionable at the moment.

CP-violation could be a key to our solving a long-standing puzzle in the Big Bang theory. Why are there atoms and molecules in the Universe? Why is there life? God, by assumption, created as much matter as antimatter. Why didn't all matter and antimatter annihilate each other in the dense infant Universe and convert into a

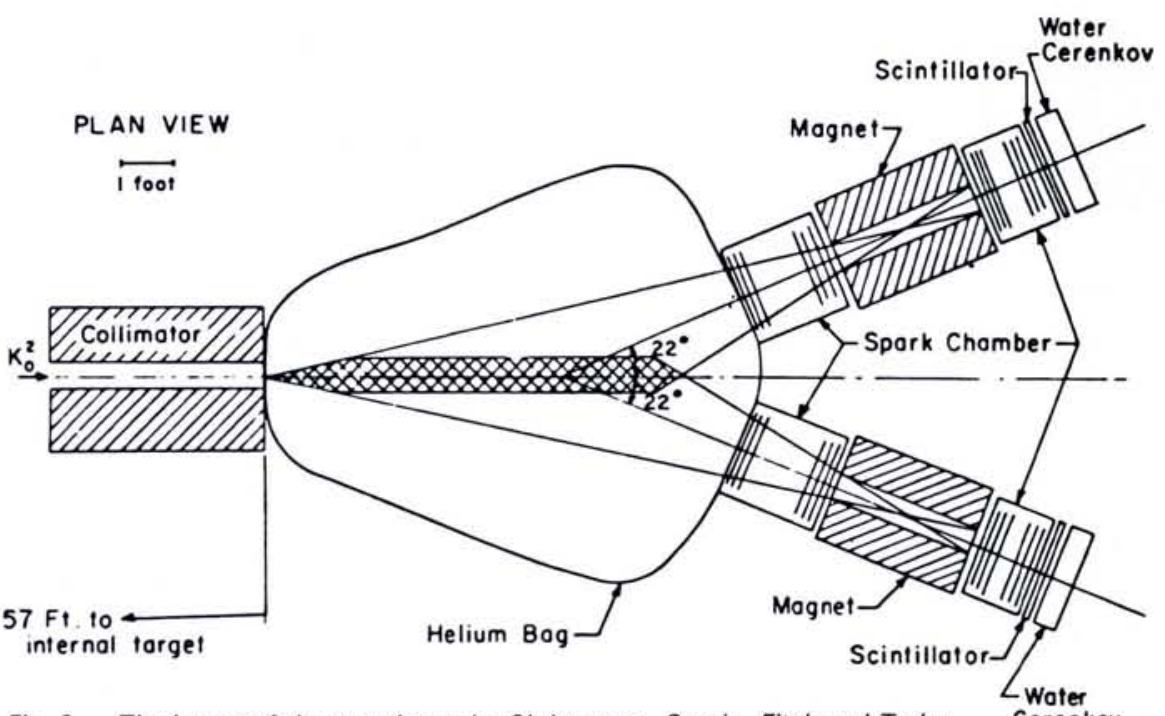

Fig. 3 - The layout of the experiment by Christenson, Cronin, Fitch and Turlay.

cloud of radiation and neutrinos/antineutrinos?

According to modern theories, there are five types of interaction at low energies: strong, electromagnetic, weak, "hyperweak" and gravitational. The hyperweak interactions are born in the process of the unification of strong and electroweak forces. Leaving aside gravity, as we go from a stronger force to a weaker, more and more symmetry principles are violated (reminiscent of Mach's principle). Weak interactions violate for example $P, C, C P$ but they respect baryon number and lepton number conservation rules. Going to hyperweak interactions, which are some 25 orders of magnitude weaker than the weak interactions, baryon number and lepton number are no longer conserved. Several experiments are under way which look for evidence of proton decay that would be a signature of the hyperweak forces.

The hyperweak forces, by violating $\mathrm{CP}$, baryon number and lepton number symmetries, generated a matter-antimatter asymmetry in the expanding newborn Universe, simply because processes producing matter, and their $\mathrm{CP}$-images producing antimatter, occurred with slightly different probabilities. As a consequence, matter exceeded antimatter by a small fraction. This tiny fraction found no antimatter to annihilate and so it remained and cooled off to give atoms, molecules and life.

The discovery of CP-violation towers as a landmark in the history of our science. James W. Cronin and Val L. Fitch are our century's Tycho Brahe and Johannes Kepler, who observed a small but deeply significant violation of a holy symmetry.

\section{New from Birkhäuser}

11th International Conference on High-Energy Accelerators

Geneva. Switzerland. July 7-11. 1980

Organized by the European Organization for Nuclear Research (CERN). Geneva

Proceedings edited by W.S. Newman. CERN, Geneva

1980. 940 pages. 200 illustrations. 100 tables. Hardcover

sFr. 174.-/DM 186,-/\$112.00

ISBN 3-7643-1215-7

Progress in Physics

Edited by Arthur Jaffe and David Ruelle

Volume 1

Pierre Collet

Jean-Pierre Eckmann

Iterated Maps on the Interval as Dynamical Systems

1980. 256 pages. Hardcover

sFr. 27.-/DM 30.-/\$14.00

ISBN 3-7643-3026-0
Volume 2

Arthur Jaffe

Clifford Taubes

Vortices and Monopoles

Structure of Static Gauge Theories

1980. 275 pages. Hardcover

SFr. 27.-/DM 30--/\$14.00

ISBN 3-7643-3025-2

Please order from your bookseller or Birkhäuser Verlag. P.O. Box 34 . $\mathrm{CH}-4010 \mathrm{Basel} /$ Switzerland or Birkhäuser Boston Inc., 380 Green Street. Cambridge MA 02139/USA 